Research Paper

\title{
A 3D Printed Porous Titanium Alloy Rod with Diamond Crystal Lattice for Treatment of the Early-Stage Femoral Head Osteonecrosis in Sheep
}

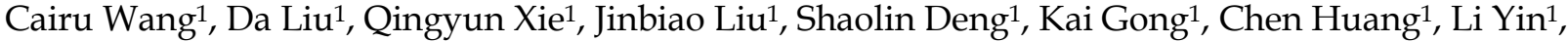 \\ Meiming $X{ }^{1}{ }^{1}$ Zheng Guo ${ }^{\bowtie}$, Wei Zheng ${ }^{\bowtie}$ \\ 1. Department of Orthopaedics, The General Hospital of Western Theater Command, Chengdu, Sichuan 610083, China \\ 2. Department of Orthopaedics, Xijing Hospital, Fourth Military Medical University, Xi'an, Shaanxi 710032, China \\ $\bowtie$ Corresponding authors: Email: guozheng@fmmu.edu.cn (Zheng Guo) and Email: zw770880@126.com (Wei Zheng) \\ (c) Ivyspring International Publisher. This is an open access article distributed under the terms of the Creative Commons Attribution (CC BY-NC) license \\ (https://creativecommons.org/licenses/by-nc/4.0/). See http://ivyspring.com/terms for full terms and conditions.
}

Received: 2018.10.21; Accepted: 2019.02.08; Published: 2019.03.09

\begin{abstract}
Instruments made of porous titanium alloy and fabricated with a 3D printed technique are increasingly used in experimental and clinical research. To date, however, few studies have assessed their use in early-stage osteonecrosis of the femoral head (ONFH). In this study, porous titanium alloy rods (Ti-Rod) with diamond crystal lattice, fabricated using an electron beam melting (EBM) technique, were implanted into sheep models $(n=9)$ of early-stage ONFH for 6 months. Bone ingrowth and integration were investigated and compared with those of sheep $(n=9)$ undergoing core decompression (CD) alone. Following Ti-Rod implantation, femoral heads showed fine osteointegration, with X-ray evaluation showing compact integration between peripheral bone and rods without radiolucent lines encircling the rods, as well as new bone growth along the metal trabeculae without the intervention of fibrous tissue. The regions of interest (ROls) of femoral heads showed fine bone ingrowth after Ti-Rod implantation than $C D$ alone. By micro-CT evaluation, the ratios of bone volume to total volume (BV/TV) of ROls in Rod group was $930 \%$ and $452 \%$ higher than $C D$ group after $3(0.206 \pm 0.0095$ vs. $0.020 \pm 0.0058, p<0.05, n=3)$ and $6(0.232$ \pm 0.0161 vs. $0.042 \pm 0.0061, p<0.05, n=3)$ months respectively. By histological evaluation, the $\mathrm{BV} / \mathrm{TV}$ of ROls in Rod group was $647 \%$ and $422 \%$ higher than CD group after $3(0.157 \pm 0.0061$ vs. $0.021 \pm 0.0061, p<0.05, n=3)$ and $6(0.235 \pm 0.0145$ vs. $0.045 \pm 0.0059, p<0.05, n=3)$ months respectively. The new bone grew along metal trabeculae into the center of the rod with a rapid bone ingrowth in Rod gorup. Whereas in CD group, new bone grew mainly at the periphery of the decompressive channel with a slow bone ingrowth. Mechanical analysis showed that maximum load on the femoral head-necks was $31 \%$ greater 6 months after Ti-Rod implantation than after CD alone when the vertical press reached the apex $(3751.75 \pm 391.96$ vs. $2858.25 \pm 512.91 \mathrm{~N}, p<0.05$, $n=3$ ). The association of rod implantation with fine bone ingrowth, osteointegration, and favorable mechanical properties suggests that implantation of the porous titanium alloy rod with the diamond crystal lattice may be a beneficial intervention for patients with early-stage ONFH.
\end{abstract}

Key words: osteonecrosis of the femoral head, porous titanium alloy, diamond crystal lattice, bone integration and ingrowth, sheep

\section{Introduction}

Osteonecrosis of the femoral head (ONFH) often affects the hips of adults aged 30-50 years [1]. If left untreated or treated non-operatively, most affected hips will progress to femoral head collapse and hip osteoarthritis [2, 3], with many of these patients requiring total hip arthroplasty (THA) procedures. As most affected patients are middle-aged, most affected hips often need prosthesis revision after THA 
procedures [4]. Surgical interventions to treat earlystage ONFH may therefore avoid or postpone THA procedures.

Surgical procedures to treat early-stage $\mathrm{ONFH}$ include standard core decompression (CD) [5, 6], multiple drilling $[7,8]$, osteotomy $[9,10]$, and $C D$ combined with adjunctive procedures [11-17]. CD has drawbacks in treating early-stage ONFH operatively, primarily because the subchondral bone loses struts after decompression. Adjunctive procedures involving CD can include bone grafts [11-14], the addition of mesenchymal cells [18-20], and tantalum rod insertion [15-17].

Because of their low elastic modulus, fine biocompatibility, and high resistance to corrosion and wear, titanium alloys are increasingly used in orthopedics and stomatology [21]. Porous titanium alloys show even lower elastic modulus while enhancing fine bone integration. 3D printing techniques, including selective laser melting (SLM) and electron beam melting (EBM), have made the process of fabricating porous titanium alloys easier. 3D printed porous titanium alloys have been utilized to treat certain conditions, such as bone tumors [22] and talar osteonecrosis [23]. However, few studies have assessed their use in large animal models of ONFH. In this study, porous titanium rods with a diamond crystal lattice were fabricated using the EBM technique and implanted into the necrotic femoral heads of sheep. Bone integration and ingrowth of these rods were investigated.

\section{Materials and Methods}

\section{Fabrication of the porous titanium alloy rods}

The parameters of the rods were based on our previous studies. The study protocol was approved by the Institutional Ethics Committee of the Fourth Military Medical University. Briefly, the femurs of small tailed Han sheep were scanned by computed tomography (CT) and the data were acquired in DICOM format. After reconstruction of all femurs, their parameters were measured. The porous titanium rods were designed to have a length of $43-51 \mathrm{~mm}$, in 2 $\mathrm{mm}$ increments, and a diameter of $6 \mathrm{~mm}$. To engage well with the lateral cortex of the femur, the $10 \mathrm{~mm}$ long end of the rod was solid; the body of the rod, which was 33-41 $\mathrm{mm}$ long in $2 \mathrm{~mm}$ increments, was porous and had a diamond crystal lattice configuration, and the tip of the rod was semispherical (Figure 1). A digital model of each rod was designed using Magic 19.0 software and exported in STL format. Each porous titanium rod was printed from Ti-6Al-4V sphere powder $(0-45 \mu \mathrm{m}$ in diameter; Xi' an Lille Metal Material Co. Ltd., China) using SLM equipment (Wuhan Binhu Mechanical and Electrical Technology Industry Co. Ltd., China). After printing, the rods were washed with an ultrasonic machine and acid solution to remove any remaining titanium alloy powder.

Four rods were scanned with Siemens micro-CT system (Inveon CT scanner, Siemens, Germany), and their average pore size and porosity were analyzed using Inveon Acquisition Workplace (IAW) (Siemens, Germany). The mean porosity of the rods was $72.70 \%$ $\pm 0.26 \%$, and their pore interconnectivity was $100 \%$. Their average pore size was $610 \pm 20 \mu \mathrm{m}$, which was similar with previous reports [24-27]. Compression strength and Young's modulus were tested using a universal mechanical testing machine (AGS $10 \mathrm{KNG}$, SHIMADZU, Japan). The direction of mechanical testing was axial which was paralleled with the axis of the rod. The mean compressive strength was $63 \pm 4.5$ $\mathrm{MPa}$, and the average Young's modulus was $2.5 \pm 0.2$ GPa.

\section{Animals and experimental design}

All procedures were strictly in accordance with the international guidelines for protection of animals, and were approved by the Animal Ethics Committee of The Fourth Military Medical University. Eighteen adult female small tailed Han sheep (age, 16-24 months; weight, 36-48 kg) were randomly divided into two groups of nine each, with one group undergoing $\mathrm{CD}$ and the other undergoing rod insertion. ONFH was induced by cryo-insult with liquid nitrogen, as described [28-30]. One month later, the sheep were performed with X-ray evaluations to confirm the presence of osteonecrosis, and then were underwent $\mathrm{CD}$ or rods insertion.

Three and six months after surgery, three animals in each group were X-rayed and sacrificed, and the operative lateral femoral heads were harvested and subjected to micro-CT scanning and histological examination (Figure 2). The remaining three animals in each group were similarly sacrificed, and the specimens were subjected to mechanical tests.

All the animals survived the operation in good physical condition, without any complications, including incision maturation, hip dislocation, or femoral neck fracture.

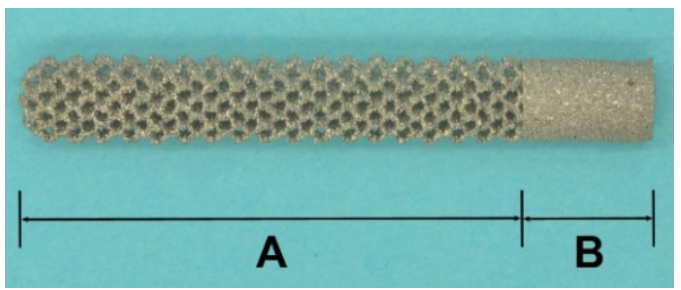

Figure 1. Photograph showing a porous titanium alloy rod with diamond crystal lattice. A: the body of the rod; B: the end of the rod. 


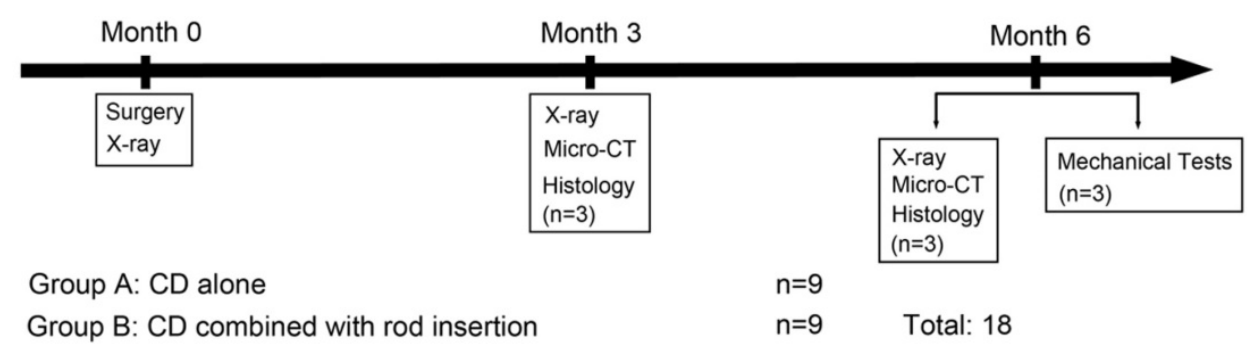

Figure 2. Experimental design of the study.

\section{Surgical procedures}

Unilateral ONFH was induced by cryo-insult as described [29, 31], with all surgical procedures performed in an aseptic operation room. The animals were fasted for 24 hours before the operation and anesthetized by intramuscular injection of xylazine hydrochloride $(0.1 \mathrm{ml} / \mathrm{kg}$; Jilin Huamu Animal Health Product Co. Ltd., China). After shaving and sterilization, a $10 \mathrm{~cm}$ incision passing through the greater trochanter was made in a posterior leg of each animal. The skin, fascia, and muscle layers were successively separated, and the posterior femoral head-neck juncture was exposed. A foramen (diameter: $2.5 \mathrm{~mm}$ ) pointed toward the center of the femoral head was made and sprayed with liquid nitrogen using cryogenic equipment (Xinxiang Xinya Cryogenic Vessel Co. Ltd., China), and the surgical field was melted using physiologic saline. The freeze/thaw cycle was repeated three times. The foramen was sealed with bone wax, and the incision was sutured in layers.

One month later, each animal was assessed by $\mathrm{X}$-ray examination to determine if ONFH had been induced successfully. When the X-ray examination showed sclerotic or cystic lesions on femoral head intervened, is was believed that the early-stage ONFH was induced. The intervened femoral heads showing sclerotic or cystic lesions without crescent sign or collapse were considered to progress early-stage ONFH. After confirmation, the CD and rod insertion procedures were performed. Anesthesia and incision were identical to those above. For sheep in both the $\mathrm{CD}$ and rod insertion groups, a hollow channel (diameter: $6.0 \mathrm{~mm}$ ) was made from the basal part of the greater trochanter and oriented to the medial-superior aspect of the femoral head. Sheep in the rod insertion group also underwent insertion of a porous titanium rod into each femoral head. After the surgery, cefazolin sodium (Sanjing Pharmaceutical Co., Ltd., $1 \mathrm{~g}$ / day) was administered intramuscularly for 48 hours to prevent infection.

\section{X-ray evaluation}

Three and six months later, three animals in each group were sacrificed, and their femoral heads were harvested and fixed in 10\% formalin solution for 72 hours. Each specimen was subjected to anteroposterior X-ray imaging. The bone ingrowth and bone integration of each image was evaluated by radiologists who were blinded to this study.

\section{Micro-CT analysis}

After X-ray examination, each specimen underwent micro-CT scanning using Siemens micro-CT system (Inveon CT scanner, Siemens, Germany) at 80 $\mathrm{kV}, 500 \mu \mathrm{A}$, and a spatial resolution of $30 \mu \mathrm{m}$. The acquired data (DICOM format) were exported and processed using Inveon Acquisition Workplace (IAW) (Siemens, Germany). A cylinder (diameter: $6.0 \mathrm{~mm}$, length: $10 \mathrm{~mm}$ ) with a hemispherical (diameter: 6.0 $\mathrm{mm}$ ) tip was defined as the region of interest (ROI), covering the decompressive channel in the $\mathrm{CD}$ group or the porous titanium rod in the rod insertion group. New bone ingrowth in the ROI of each specimen was reconstructed with the threshold of 200-1400 for bone and 1400-4095 for implant, and the ratio of new bone volume to total volume (BV/TV) and the mean bone trabecular thickness in each ROI were calculated.

\section{Histological examination}

After micro-CT scanning, the specimens were dehydrated and embedded in methylmethacrylate. Each specimen was divided into six representative sections in the coronal plane, with the two middle sections selected as ROIs. Two $300 \mu \mathrm{m}$ slices were cut form each selected section using a microtome (Leica, Germany). After grinding and polishing, the slices were mounted on microscope slides and stained with Van Gieson's picrofuchsin. The tissues stained with red color was the bone, and the metal trabeculae could not be stained and was light-proof. The qualitative analysis of BV/TV of ROIs was performed with a light microscope (Leica LA Microsystems, Germany) and calculated by Image-Pro Plus software (Silver Spring, USA).

\section{Mechanical test}

At 6 months, three animals in each group were sacrificed, and their femurs were harvested and stored at $-80^{\circ} \mathrm{C}$. The distal $1 / 3$ of each femur was 
resected and fixed onto a self-designed mechanical clamping apparatus, which engaged with a universal mechanical testing machine (AGS 10 KNG, SHIMADZU, Japan). The femur was kept in a physiological standing position by adjusting the clamping apparatus. The physiological position was defined that the femur has an adduction angle of 9.6 degree, and the femur axis was set paralleled with coronal plane. A pressor probe was designed to press the femoral head, which had a concave spherical shape tangent to the femoral head. Each femoral head was applied to a vertical press with a speed of 2 $\mathrm{mm} / \mathrm{min}$, and each press-displacement curve was drawn according to primary data.

\section{Statistical analysis}

Quantitative data were expressed as means \pm standard deviation and compared using LSD t-tests. All statistical analyses were performed using SPSS 16.0 software (International Business Machines Corporation, Armonk, USA), with $p$-values < 0.05 considered statistically significant.

\section{Results}

\section{X-ray evaluation}

None of the animals experienced flattened femoral heads, femoral neck fractures, or rod displacements. At 3 and 6 months, all the decompressive channels of the femoral heads in the CD group were radiolucent. At 6 months, the size of radiolucent area was smaller than 3 months showing a slow bone ingrowth process. Whereas in rod group, there was no obvious radiolucent line between the rod and peripheral bone at 3 and 6 months, indicating a rapid bone ingrowth and fine osteointegration.

\section{Micro-CT analysis}

Cross-section images showed that all decompressive channels in the $\mathrm{CD}$ group remained unclosed at 3 (Figure 4B) and 6 (Figure 4C) months. The diameter of the decompressive channel became narrower over time, indicating a slow process of bone ingrowth. By contrast, new bone had grown into the center of the rod at 3 months (Figure 4E) and grew further into the rods at 6 months (Figure 4F) in the rod insertion group.
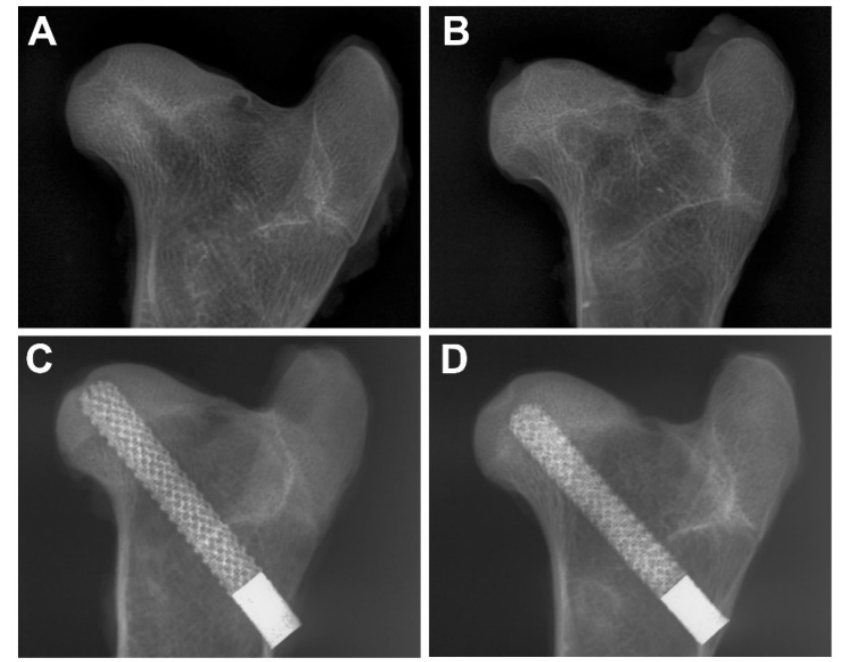

Figure 3. Post-operative $X$-ray evaluations. The decompressive channels were radiolucent in the $C D$ group at $3(A)$ and 6 (B) months, whereas the porous titanium rods showed fine osteointegration at $3(C)$ and $6(D)$ months.
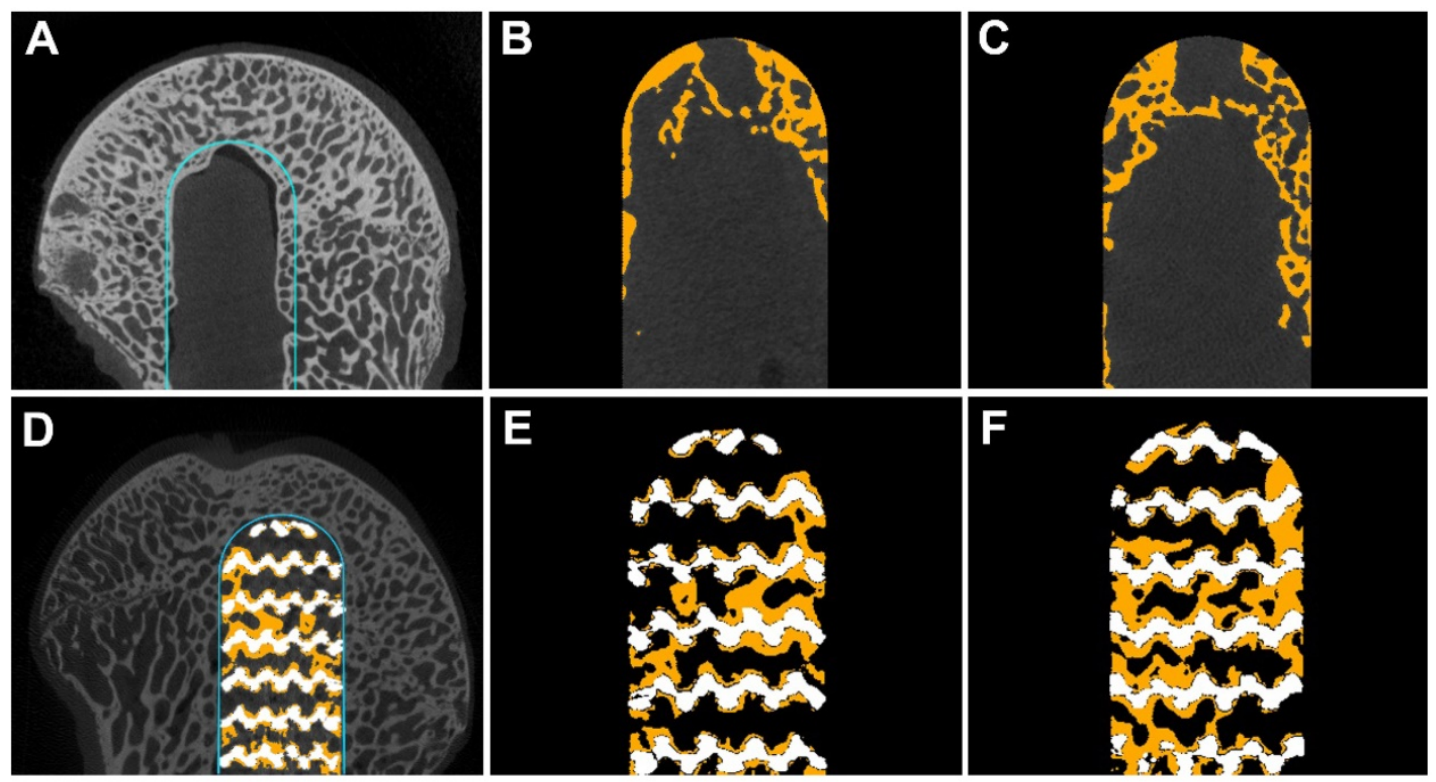

Figure 4. Cross-sectional images on micro-CT examination. In the ROI of the CD group (blue line) (A), new bone (yellow) grew mainly at the periphery of the decompressive channel at 3 (B) and 6 (C) months. By contrast, in the ROI of the rod insertion group (blue line) (D), the new bone grew along the metal trabeculae into the center of the rod at $3(E)$ and $6(F)$ months. 
At 3 months, the BV/TV of ROIs in Rod group was $930 \%$ higher than CD group (0.206 \pm 0.0095 vs. $0.020 \pm 0.0058, p<0.05, \mathrm{n}=3$ ), while the mean trabecular thickness of ROIs in Rod group was $52 \%$ lower than CD group $(0.087 \pm 0.0119$ vs. $0.184 \pm 0.0088$ $\mathrm{mm}, p<0.05, \mathrm{n}=3)$. At 6 months, the BV/TV of ROIs in Rod group was $452 \%$ higher than CD group (0.232 \pm 0.0161 vs. $0.042 \pm 0.0061, p<0.05, n=3)$, and the mean trabecular thickness of ROIs in Rod group was $26 \%$ lower than CD group (0.142 \pm 0.0162 vs. $0.193 \pm 0.0074$ $\mathrm{mm}, p<0.05, \mathrm{n}=3$ ). (Figures 5 and 6 )

\section{Histological analysis}

At 3 months, new bone in the $\mathrm{CD}$ group was observed to grow from the periphery to the center of the decompressive channel, with the channel remaining unclosed (Figure 7A). At 6 months, the decompressive channel remained unclosed in the $\mathrm{CD}$ group (Figure 7B), but its diameter decreased, indicating a slow process of bone ingrowth. In the rod insertion group, newly formed bone at 3 months was mainly present on the periphery of the rod (Figure 7C), with no new bone at its center. At 6 months, new bone had grown further into the center of the rod (Figure 7D).

Analysis of these images using Image-Pro Plus software showed that the BV/TV of ROIs was $647 \%$ $422 \%$ higher in Rod group than CD group at $3(0.157$ \pm 0.0061 vs. $0.021 \pm 0.0061, p<0.05, \mathrm{n}=3)$ and $6(0.235 \pm$ 0.0145 vs. $0.045 \pm 0.0059, p<0.05, \mathrm{n}=3$ ) months respectively (Figure 8).

\section{Mechanical test}

When subjected to a vertical press, each specimen showed a moderate deformity and a fracture of the femoral head-neck juncture. Each specimen had a single and similar load-displacement curve, but the slopes were higher in the rod insertion than in the CD group. After a gentle slope about 0.4 $\mathrm{mm}$ displacement in the axis, the curve became more precipitous and finally reached its apex, which was characterized as the femoral head-neck fracture during the test. The maximum load of Rod group was $31 \%$ greater than $\mathrm{CD}$ group $(2858.25 \pm 512.91$ vs. $3751.75 \pm 391.96 \mathrm{~N}, p<0.05, \mathrm{n}=3$, Figure 9).

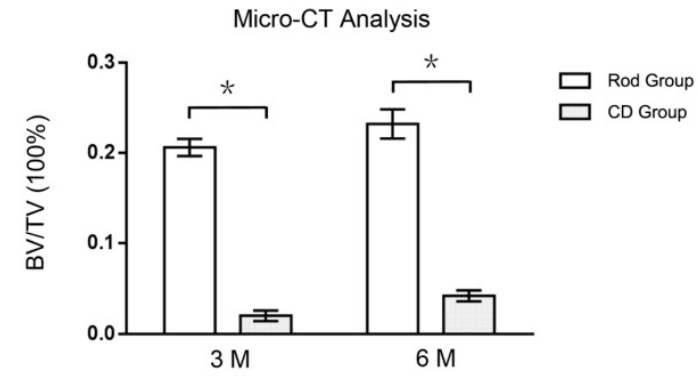

Figure 5. Ratio of bone volume to total volume (BV/TV) of ROI in the rod insertion and CD groups, as determined by micro-CT analysis. Asterisks $\left({ }^{*}\right)$ indicate statistical significance $(p<0.05)$.

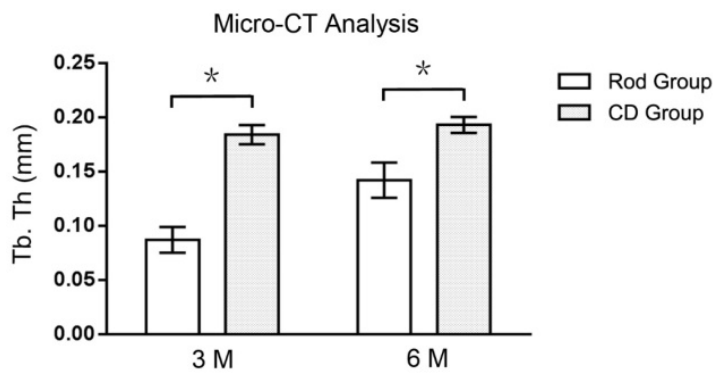

Figure 6. The mean trabecular thickness of the ROI of the rod insertion and CD groups, as determined by micro-CT analysis. Asterisks $(*)$ indicate statistical significance $(p<0.05)$.

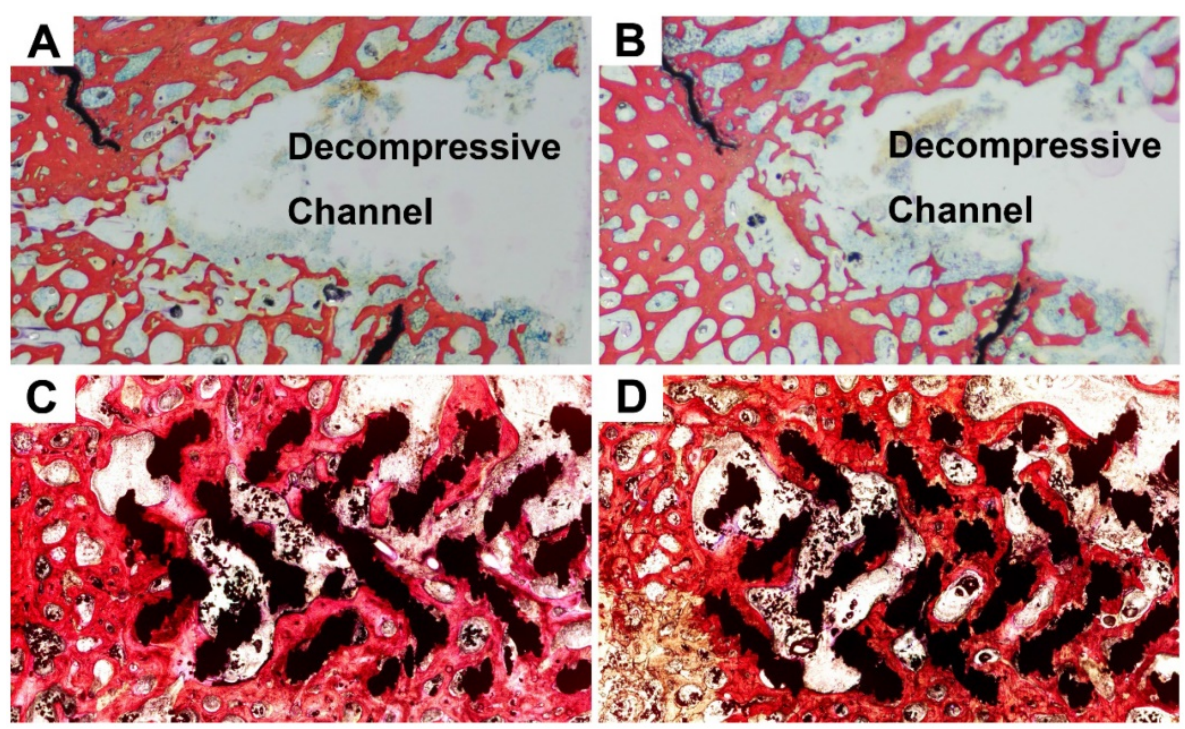

Figure 7. Histological images of the ROI of the specimens of the $C D(A, B)$ and rod insertion (C,D) groups at 3 (A,C) and 6 (B,D) months. In the $C D$ group, new bone (red trabeculae) grew slowly from the periphery of the decompressive channel to the center at $3(A)$ and 6 (B) months. In the rod insertion group, the new bone (red trabeculae) grew along the metal trabeculae (black), with fine osteointegration, at 3 (C) and 6 (D) months. 


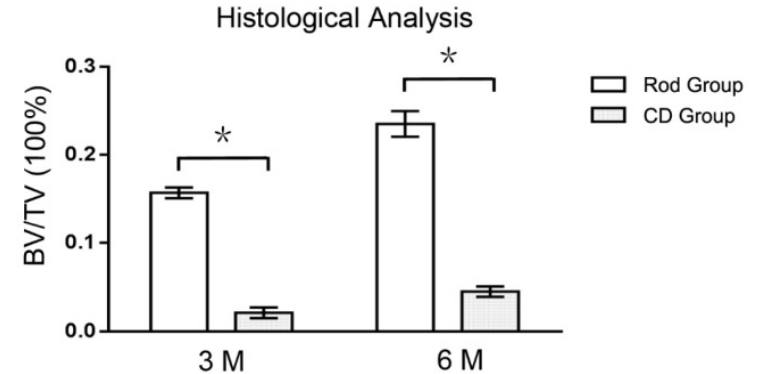

Figure 8. Histological analysis of bone volume to total volume (BV/TV) ratios of ROls of the CD and rod insertion groups at 3 and 6 months. Asterisks $\left(^{*}\right)$ indicate statistical significance $(p<0.05)$.

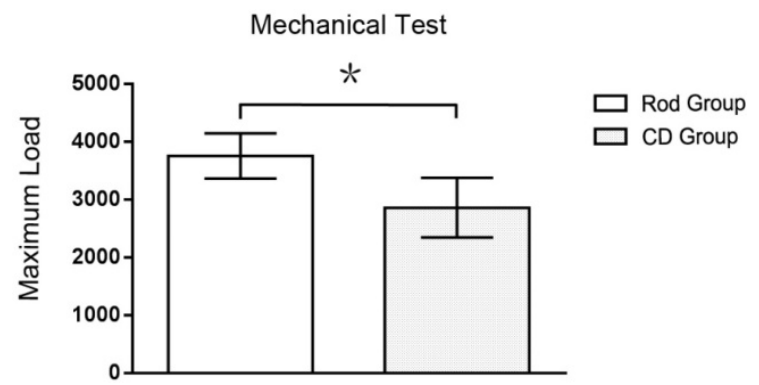

Figure 9. The maximum loads in mechanical tests of the two groups. Asterisk $(*)$ indicates statistical significance $(p<0.05)$

\section{Discussion}

During early-stage ONFH, the subchondral bone of the femoral head often loses its mechanical strut after $\mathrm{CD}$ alone. A bone graft, whether vascularized or non-vascularized, can provide mechanical support for the subchondral bone and increase the survival of the affected femoral heads[2, 32]. However, the combination of $\mathrm{CD}$ and bone-graft implantation is technically difficult[33] and associated with donor site morbidity [34]. A porous titanium alloy can be utilized for mechanical strut after CD during early-stage ONFH. The high porosity and moderate pore size of the alloy can promote bone ingrowth and fine osteointegration [35]. In addition, the ideal elastic modulus of the implant, which is similar to host bone, can prevent the effects of stress shielding, reducing the risk of implant loosening. In 2018, Ying Zhang et al. reported a 3D printed titanium rod implanted in human early-stage ONFH [36]. With 24 months follow-up, the authors concluded that this titanium rod can get a satisfactory treatment effect on ONFH of ARCO IIA and ARCO IIB. Unlike clinical results of aforementioned study[36], our research mainly focused on histological and radiological changes of sheep early-stage ONFH intervened with a porous titanium rod. Meanwhile, the pore structure of porous titanium alloy was also described detailed in this study.

The micro-architecture of porous titanium alloys can be classified as closed and open cells. Closed cells, which are often fabricated as a thin wall or membrane enclosed within the solid metal, are mainly used in weight bearing implants, such as acetabulum cups and femoral stems. By contrast, bones of femoral head and neck are primarily cancellous. After standard CD procedures, the decompressive channel often requires an ideal implant with lower elastic modulus to avoid stress shielding and a porous structure to enhance fine bone ingrowth and osteointegration. Porous titanium alloy rods with open-cell units may therefore be suitable. Traditional methods of manufacturing open cells include powder metallurgy, sintering of powders or fibers, electro-deposition, and vapor deposition [37]. These fabricating processes are complicated. Although pore size and structure can be adjusted by changing the fabricating parameters, only a few specified porous micro-architectures may be available. It is difficult to manufacture open-cell porous titanium alloy implants with precisely controlled pore structure, pore size, and elastic modulus using these methods.

Metal-based 3D printing techniques, including SLM and EBM, have made the process of fabricating open-cell porous titanium alloys easier. The mechanical properties of these alloys were similar to those of conventional porous titanium alloys[38, 39]. Computer aided design (CAD) and image-based design can be utilized in digital models of 3D printing techniques[35]. CAD design often involves software, such as Magics (Materialise, Belgium) or Unigraphics NX (Siemens PLM Software, Germany). Inputting the digital model into the 3D printing equipment can easily yield an ideal porous titanium alloy scaffold or rod with pre-designed micro-architecture. A commonly used open-cell unit of porous titanium alloy is a diamond crystal lattice[25-27, 40,41], which, in animal models, has been found to promote fine bone ingrowth and osteointegration in necrotic talus and intervertebral spaces. These findings suggested that this porous titanium alloy with a diamond crystal lattice may be a candidate implant for early-stage ONFH after standard CD procedures.

Based on CT scans of sheep femurs, we designed porous titanium rods with the aforementioned diamond crystal lattice and tested the effect of these rods on bone ingrowth and osteointegration in a sophisticated model in sheep[30, 31]. X-rays evaluating general condition and bone ingrowth found no complications, such as hip dislocation and femoral neck fracture, and no incidence of femoral head collapse in both the $C D$ and rod insertion groups. Until 6 months, the decompressive channel was still radiolucent in the $\mathrm{CD}$ group, showing that bone restoration was sluggish, a finding confirmed by micro-CT evaluation. New bone in this group grew 
slowly from the periphery to the center of the decompressive channel at 3 and 6 months. In the rod insertion group, however, there was no obvious radiolucent line between the rod and peripheral bone, suggesting early bone ingrowth. Micro-CT examination detected new bone growing along the metal trabeculae at 3 and 6 months. The immediate structural and mechanical restoration of subchondral bone of a necrotic femoral head is vital after the CD procedure. If it loses mechanical support, the decompressive femoral head can easily progress to collapse. Although none of the femoral heads in the $\mathrm{CD}$ group collapsed, this may have been due to differences between sheep and humans in the weight bearing of the femoral head.

In quantitative analysis, we selected the some ROI for each head and compared the BV/TVs of the two groups. This ratio was significantly higher in the rod insertion than in the CD group at 3 and 6 months, as shown by both micro-CT and histological evaluation. The higher BV/TV in the rod insertion group indicated more new bone formation. In Rod group, the new bone grew along metal trabeculae into the center of the rod with a rapid ingrowth evidenced by micro-CT and histological evaluation at 3 months. Whereas in CD group, the now bone grew mainly at the periphery of the decompressive channel with a slow bone ingrowth evidenced by micro-CT and histological evaluation at 6 months. In micro-CT evaluation, mean trabecular thickness was significantly lower in the rod insertion than in the CD group, perhaps because the new bone that grew along the metal trabeculae in the rod insertion group was thinner than the new bone that grew into the decompressive channel in the $\mathrm{CD}$ group. Further investigations are needed to understand these mechanisms of bone growth.

As we known, bone remodeling process is predominated by a dynamic balance between osteoclastic bone resorption and osteoblastic bone formation[42]. This process is affected by peripheral mechanical and biochemical environment of the bone. In this study, the rapid bone ingrowth in Rod group might be attributed to its predominant bone formation comparing to $\mathrm{CD}$ group. However, its molecular mechanism is unclear. The pathways involved in bone remodeling include Wnt, TGF- $\beta$ /BMPk, RANKL-OPG et al. [42]. Wnt pathway plays an important role in bone remodeling process $[43,44]$. Also, previous studies $[45,46]$ had reported that surface modification and local mechanical stimulation can improve osteointegration via Wnt pathway. The relationship between Wnt pathway and necrotic femoral heads intervened by this porous titanium alloy rods need further investigation.
Previously, it has been shown that quadrupeds, including sheep and dogs, often fail to progress to femoral head collapse [30, 47, 48]. By contrast, emus, which are large, ostrich-like bipeds native to Australia, can progress to femoral head collapse following ischemic or cryogenic insults[31, 49]. Animal models of ONFH induced by cryogenic or absolute ethanol insults have shown histological and imaging changes similar to those of early-stage human ONFH [30, 47, 48]. Necrotic femoral heads in these animal models following standard CD procedures or implantation of porous titanium alloy rods can theoretically mimic the histological and imaging changes observed in human $\mathrm{ONFH}$, suggesting that these models can provide clues to new treatments for early-stage human ONFH.

Mechanical tests at maximum axial load did not result in femoral head collapse rather than fracture at the femoral head-neck juncture, a finding that may also be attributed to differences in the mechanical environment of the hips between quadrupeds and bipeds. High biomechanical challenge in both can overwhelm osteolytic repair processes, causing the femoral head to collapse[31]. Because collapse is gradual, blood circulation in the femoral heads of bipeds can undergo further degeneration. In quadrupeds, however, the temperate biomechanical environment can allow slow repair of necrotic bone or areas of intervention. Thus, the mechanical properties of femoral heads in sheep models can be maintained. In response to vertical pressure, these femoral heads did not collapse rather than undergoing fracture at the femoral head-neck juncture.

One of the limitations of this study was that the observational period was only six months. Histological, imageological and mechanical changes over six months need further investigation. Another limitation was that the animals used in this study were sheep which were quadrupeds rather than bipeds. Theoretically, hip mechanical environment of bipeds was more similar to humans. There have been sophisticated animal model of emus reported [31, 50]. Further researches can be tried using emu models.

\section{Conclusion}

Sheep models of early-stage ONFH intervened with porous titanium alloy rod with the diamond crystal lattice showed fine bone ingrowth, osteointegration, and favorable mechanical properties, which suggested that this porous titanium rod may be a beneficial intervention for patients with early-stage ONFH. Further clinical researches need to be carried out.

\section{Acknowledgements}

This work was supported by the National Key 
R\&D Program of China (2017YFC1104900), the National Natural Science Foundation of China (No. 811717 73), the National Natural Science Foundation of China (No. 5150010028), and the Projects of Science and Technology of Sichuan Province (No. 2019YFS0122).

\section{Competing Interests}

The authors have declared that no competing interest exists.

\section{References}

[1] Mont MA, Hungerford DS. Non-traumatic avascular necrosis of the femoral head. J Bone Joint Surg Am. 1995;77:459.

[2] Mont MA, Cherian JJ, Sierra RJ, et al. Nontraumatic Osteonecrosis of the Femoral Head: Where Do We Stand Today?: A Ten-Year Update. J Bone Joint Surg Am. 2015;97:1604-27.

[3] Mont MA, Jones LC, Hungerford DS. Nontraumatic osteonecrosis of the femoral head: ten years later. J Bone Joint Surg Am. 2006;88:1117-32.

[4] Park YS, Moon YW, Lee KH, et al. Revision hip arthroplasty in patients with a previous total hip replacement for osteonecrosis of the femoral head. Orthopedics. 2014;37:e1058-62.

[5] Sadile F, Bernasconi A, Russo S, et al. Core decompression versus other joint preserving treatments for osteonecrosis of the femoral head: a meta-analysis. Br Med Bull. 2016;118:33-49.

[6] Pierce TP, Jauregui JJ, Elmallah RK, et al. A current review of core decompression in the treatment of osteonecrosis of the femoral head. Curr Rev Musculoskelet Med. 2015:8:1-5.

[7] Omran AA. Multiple drilling compared with standard core decompression for avascular necrosis of the femoral head in sickle cell disease patients. Arch Orthop Trauma Surg. 2013;133:609-13.

[8] Ji YB, Dai SK, Park KS, et al. Finite element analysis of the multiple drilling technique for early osteonecrosis of the femoral head. Ann Biomed Eng. 2013;41:2528-37

[9] Yamamoto T, Motomura G, Karasuyama K, et al. Results of the Sugioka transtrochanteric rotational osteotomy for osteonecrosis: Frequency and role of a defect of the quadratus femoris muscle in osteonecrosis progression. Orthop Traumatol Surg Res. 2016;102:387-90.

[10] Zhao G, Yamamoto T, Ikemura S, et al. Radiological outcome analysis of transtrochanteric curved varus osteotomy for osteonecrosis of the femoral head at a mean follow-up of 12.4 years. J Bone Joint Surg Br. 2010;92:781-6.

[11] Shah SN, Kapoor CS, Jhaveri MR, et al. Analysis of outcome of avascular necrosis of femoral head treated by core decompression and bone grafting. J Clin Orthop Trauma. 2015;6:160-6.

[12] Pierce TP, Elmallah RK, Jauregui JJ, et al. A current review of non-vascularized bone grafting in osteonecrosis of the femoral head. Curr Rev Musculoskelet Med. 2015;8:1-6

[13] Cesar GFPD, Roca I, Barber I, et al. Femoral head bone viability after free vascularized fibular grafting for osteonecrosis: SPECT/CT study. Microsurgery. 2016;36:573-7.

[14] Gao YS, Chen SB, Jin DX, et al. Modified surgical techniques of free vascularized fibular grafting for treatment of the osteonecrosis of femoral head: Results from a series of 407 cases. Microsurgery. 2013;33:646-51.

[15] J M, W S, F G, et al. Porous Tantalum Implant in Treating Osteonecrosis of the Femoral Head: Still a Viable Option? Sci Rep. 2016;6:28227.

[16] Pakos EE, Megas P, Paschos NK, et al. Modified porous tantalum rod technique for the treatment of femoral head osteonecrosis. World J Orthop. 2015;6:829.

[17] Liu Y, Liang Y, Zhou S, et al. Tantalum rod implantation for femoral head osteonecrosis: survivorship analysis and determination of prognostic factors for total hip arthroplasty. Int Orthop. 2015:1-11.

[18] $\mathrm{Li} \mathrm{X}, \mathrm{Xu}$ X, Wu W. Comparison of bone marrow mesenchymal stem cells and core decompression in treatment of osteonecrosis of the femoral head: a meta-analysis. Int J Clin Exp Pathol. 2014;7:5024-30.

[19] Zhao D, Cui D, Wang B, et al. Treatment of early stage osteonecrosis of the femoral head with autologous implantation of bone marrow-derived and cultured mesenchymal stem cells. Bone. 2012;50:325.

[20] Gangii V, Hauzeur JP, Matos C, et al. Treatment of osteonecrosis of the femoral head with implantation of autologous bone-marrow cells. A pilot study. J Bone Joint Surg Am. 2004:86-A:1153-60.

[21] Geetha M, Singh A, Asokamani R, et al. Ti based biomaterials, the ultimate choice for orthopaedic implants-a review. Prog Mater Sci. 2009;54:397-425.

[22] Fan $\mathrm{H}, \mathrm{Fu} \mathrm{J}, \mathrm{Li} \mathrm{X}$, et al. Implantation of customized 3-D printed titanium prosthesis in limb salvage surgery: a case series and review of the literature. World J Surg Oncol. 2015;13:1-10.

[23] Yuan C, Wang J, Zhang Y, et al. Development of a clinically relevant animal model for the talar osteonecrosis in sheep. Int J Med Sci. 2012:9:816.

[24] Li Y, Yang W, Li X, et al. Improving Osteointegration and Osteogenesis of Three-Dimensional Porous Ti6Al4V Scaffolds by Polydopamine-Assisted
Biomimetic Hydroxyapatite Coating. Acs Applied Materials \& Interfaces. 2015;7:5715-24.

[25] Wu S, Li Y, Zhang Y, et al. Porous titanium-6 aluminum-4 vanadium cage has better osseointegration and less micromotionthan a poly-ether-ether-ketone cage in sheep vertebral fusion. Artif Organs. 2013;37:E191-E201.

[26] Taniguchi N, Fujibayashi S, Takemoto M, et al. Effect of pore size on bone ingrowth into porous titanium implants fabricated by additive manufacturing: An in vivo experiment. Mater Sci Eng C Mater Biol Appl. 2016;59:690-701.

[27] Hara D, Nakashima $Y$, Sato $T$, et al. Bone bonding strength of diamond-structured porous titanium-alloy implants manufactured using the electron beam-melting technique. Mater Sci Eng C Mater Biol Appl. 2016;59:1047-52

[28] Yang S, Wu X, Mei R, et al. Biomaterial-loaded allograft threaded cage for the treatment of femoral head osteonecrosis in a goat model. Biotechnol Bioeng. 2008;100:560-6.

[29] Tang T, Lu B, Yue B, et al. Treatment of osteonecrosis of the femoral head with hBMP-2-gene-modified tissue-engineered bone in goats. J Bone Joint Surg Br. 2007:89:127-9.

[30] Velez R, Soldado F, Hernandez A, et al. A new preclinical femoral head osteonecrosis model in sheep. Arch Orthop Trauma Surg. 2011;131:5-9.

[31] Conzemius MG, Brown TD, Zhang Y, et al. A new animal model of femoral head osteonecrosis: one that progresses to human-like mechanical failure. J Orth Res. 2002;20:303-9.

[32] Chughtai M, Piuzzi NS, Khlopas A, et al. An evidence-based guide to the treatment of osteonecrosis of the femoral head. Bone \& Joint Journal. 2017;99-B:1267.

[33] Marker DR, Seyler TM, McGrath MS, et al. Treatment of early stage osteonecrosis of the femoral head. J Bone Joint Surg Am. 2008:90 Suppl 4:175-87.

[34] Zalavras CG, Lieberman JR. Osteonecrosis of the femoral head: evaluation and treatment. J Am Acad Orthop Surg. 2014;22:455-64.

[35] Wang X, Xu S, Zhou S, et al. Topological design and additive manufacturing of porous metals for bone scaffolds and orthopaedic implants: A review. Biomaterials. 2016;83:127.

[36] Zhang, Ying, Zhang, et al. A new 3D printed titanium metal trabecular bone reconstruction system for early osteonecrosis of the femoral head. Medicine. 2018;97:e11088.

[37] Ryan G, Pandit A, Apatsidis DP. Fabrication methods of porous metals for use in orthopaedic applications. Biomaterials. 2006;27:2651-70.

[38] Marin E, Fusi S, Pressacco M, et al. Characterization of cellular solids in Ti6Al4V for orthopaedic implant applications: Trabecular titanium. Journal of the Mechanical Behavior of Biomedical Materials. 2010;3:373-81.

[39] Parthasarathy J, Starly B, Raman S, et al. Mechanical evaluation of porous titanium (Ti6Al4V) structures with electron beam melting (EBM). J Mech Behav Biomed Mater. 2010:3:249-59.

[40] Li X, Yuan C, Wang J, et al. The treatment effect of porous titanium alloy rod on the early stage talar osteonecrosis of sheep. PLoS One. 2013;8:e58459.

[41] Ahmadi SM, Campoli G, Amin YS, et al. Mechanical behavior of regular open-cell porous biomaterials made of diamond lattice unit cells. J Mech Behav Biomed Mater. 2014;34:106.

[42] Wang Y, Li Y, Paulson C, et al. Wnt and the Wnt signaling pathway in bone development and disease. Front Biosci (Landmark Ed). 2014;19:379-407.

[43] Karner CM, Long F. Wnt signaling and cellular metabolism in osteoblasts. Cell Mol Life Sci. 2017;74:1649-57.

[44] Duan P, Bonewald LF. The role of the wnt/ $\beta$-catenin signaling pathway in formation and maintenance of bone and teeth. Int $J$ Biochem Cell Biol. 2016;77:23

[45] Liu W, Chen D, Jiang G, et al. A lithium-containing nanoporous coatings on entangled titanium scaffold can enhance osseointegration through Wnt/ $\beta$-catenin pathway. Nanomedicine. 2018;14:153

[46] Jing D, Tong S, Zhai M, et al. Effect of low-level mechanical vibration on osteogenesis and osseointegration of porous titanium implants in the repair of long bone defects. Sci Rep. 2015;5:17134.

[47] Wang C, Wang J, Zhang Y, et al. A canine model of femoral head osteonecrosis induced by an ethanol injection navigated by a novel template. Int J Med Sci. 2013;10:1451-8.

[48] Manggold J, Sergi C, Becker K, et al. A new animal model of femoral head necrosis induced by intraosseous injection of ethanol. Lab Anim. 2002;36:173-80.

[49] Goetz JE, Robinson DA, Pedersen DR, et al. Cryoinsult parameter effects on the histologically apparent volume of experimentally induced osteonecrotic lesions. J Orth Res. 2011;29:931-7.

[50] Zheng L, Liu Z, Lei M, et al. Steroid-associated hip joint collapse in bipedal emus. PLoS One. 2013;8:e76797. 